\title{
Host control of Mycobacterium tuberculosis is regulated by 5 -lipoxygenase-dependent lipoxin production
}

\author{
Andre Bafica, ${ }^{1,2}$ Charles A. Scanga, ${ }^{1}$ Charles Serhan, ${ }^{3}$ Fabiana Machado, ${ }^{4}$ Sandy White, ${ }^{1}$ \\ Alan Sher, ${ }^{1}$ and Julio Aliberti ${ }^{4}$ \\ ${ }^{1}$ Immunobiology Section, Laboratory of Parasitic Diseases, National Institute of Allergy and Infectious Diseases (NIAID), NIH, Bethesda, Maryland, USA. \\ 2Laboratorio de Imunorregulacao e Microbiologia, Centro de Pesquisas Goncalo Moniz, Fundação Oswaldo Cruz, Salvador, Bahia, Brazil. \\ ${ }^{3}$ Center for Experimental Therapeutics and Reperfusion Injury, Department of Anesthesiology, Perioperative and Pain Medicine, \\ Brigham and Women's Hospital, Harvard Medical School, Boston, Massachusetts, USA. "Department of Immunology, \\ Duke University Medical School, Durham, North Carolina, USA.
}

\begin{abstract}
Th1 type cytokine responses are critical in the control of Mycobacterium tuberculosis infection. Recent findings indicate that 5-lipoxygenase-dependent (5-LO-dependent) lipoxins regulate host IL-12 production in vivo. Here, we establish lipoxins as key chemical mediators in resistance to $M$. tuberculosis infection. High levels of lipoxin $\mathrm{A}_{4}\left(\mathrm{LXA}_{4}\right)$ were detected in sera from infected WT but not infected 5-LO-deficient mice. Moreover, lungs from M. tuberculosis-infected 5- $\mathrm{lo}^{-/-}$animals showed increased IL-12, IFN- $\gamma$, and NO synthase 2 (NOS2) mRNA levels compared with the same tissues in WT mice. Similarly, splenocyte recall responses were enhanced in mycobacteria-infected 5- $l o^{-/-}$versus WT mice. Importantly, bacterial burdens in 5-lo-/- lungs were significantly lower than those from WT mice, and this enhancement in the resistance of the 5-lo-/- animals to M. tuberculosis was completely prevented by administration of a stable $\mathrm{LXA}_{4}$ analog. Together our results demonstrate that lipoxins negatively regulate protective Th1 responses against mycobacterial infection in vivo and suggest that the inhibition of lipoxin biosynthesis could serve as a strategy for enhancing host resistance to $M$. tuberculosis.
\end{abstract}

\section{Introduction}

Th1-mediated immunity plays a crucial role in host defense against Mycobacterium tuberculosis. Cytokines such as IL-12, IFN- $\gamma$, and TNF are essential for protection against this pathogen in the mouse model (1-3). Additional evidence suggests that the same cytokines are important resistance factors in the human immune response against mycobacterial infection (4-7). In addition to the Th1 type response mounted over the course of infection, downregulatory mediators may be important players in controlling excessive synthesis of proinflammatory cytokines and subsequent tissue damage and could contribute to the promotion of bacterial survival. Nevertheless, Th2 cytokines such as IL-4 and IL-13 have been described as playing no or only a limited role in in vivo M. tuberculosis infection (8-10). Similarly, although in vitro IL-10 production is associated with reduced human disease (11), mice deficient in this important downregulatory cytokine show nearly normal control of $M$. tuberculosis infection $(9,10)$.

There is a growing body of evidence indicating that a class of lipoxygenase-derived eicosanoids known as lipoxins plays an important role in the immunoregulation of inflammation-associated disease (12). We have previously shown that lipoxin $\mathrm{A}_{4}\left(\mathrm{LXA}_{4}\right)$, a lipid mediator derived locally from 5-lipoxygenase (5-LO) biosynthetic pathways, acts in vitro as a negative regulator of DC IL-12 production triggered by the intracellular protozoan parasite Toxoplasma gondii (13). An in vivo role for this pathway in host resistance to the same pathogen

Nonstandard abbreviations used: $\mathrm{LTB}_{4}$, leukotriene $\mathrm{B}_{4}$; 5-LO, 5-lipoxygenase; $\mathrm{LXA}_{4}$, lipoxin $\mathrm{A}_{4}$; NOS2, NO synthase 2.

Conflict of interest: The authors have declared that no conflict of interest exists.

Citation for this article: J. Clin. Invest. 115:1601-1606 (2005).

doi:10.1172/JCI23949. was suggested by the observation that T. gondii-infected 5-LO-deficient mice succumb as a result of exacerbated proinflammatory responses despite diminished parasite numbers (14).

In the present report, we asked whether 5-LO-dependent mechanisms, and in particular those mediated by lipoxins, also play a role in regulating host resistance to $M$. tuberculosis. To do so, we examined the course of infection- and pathogen-induced cellular immune responses in 5-LO-deficient mice exposed to mycobacteria by aerosol exposure. Our results reveal a major role for 5-LO-dependent lipoxin synthesis in the immune modulation of $M$. tuberculosis infection in vivo and suggest that this pathway may be a potential target for therapeutic intervention in tuberculosis.

\section{Results}

M. tuberculosis-infected mice produce $\mathrm{LXA}_{4}$ in a 5-LO-dependent manner. To assess whether 5-LO plays a role in $M$. tuberculosis infection in vivo, we first measured its products leukotriene $\mathrm{B}_{4}\left(\mathrm{LTB}_{4}\right)$ and $\mathrm{LXA}_{4}$ in sera from $\mathrm{B} 6,129 \mathrm{~S} \mathrm{~F} 2 / \mathrm{J}$ mice at different time points after aerosol infection (300 CFU/animal). As shown in Figure 1, these eicosanoids were detected at high levels as early as 1 week after M. tuberculosis infection. $\mathrm{LXA}_{4}$, but not $\mathrm{LTB}_{4}$, synthesis was maintained during chronic infection. Importantly, neither eicosanoid was detected above background levels in M. tuberculosis-infected 5-lo-/- animals, which confirmed the dependence of $\mathrm{LTB}_{4}$ and $\mathrm{LXA}_{4}$ on 5-LO in vivo (Figure 1, A and B). To address the issue of which cell population is responsible for 5-LO activity, we performed immunostaining for the enzyme in lung sections of $M$. tuberculosisinfected WT mice. 5-LO-positive staining was found to colocalize with endothelium (Figure 1C) and F4/80+ cells (Figure 1D). Taken together, these results indicate that $\mathrm{LTB}_{4}$ and $\mathrm{LXA}_{4}$ are strongly induced during $M$. tuberculosis infection in vivo and suggest that 

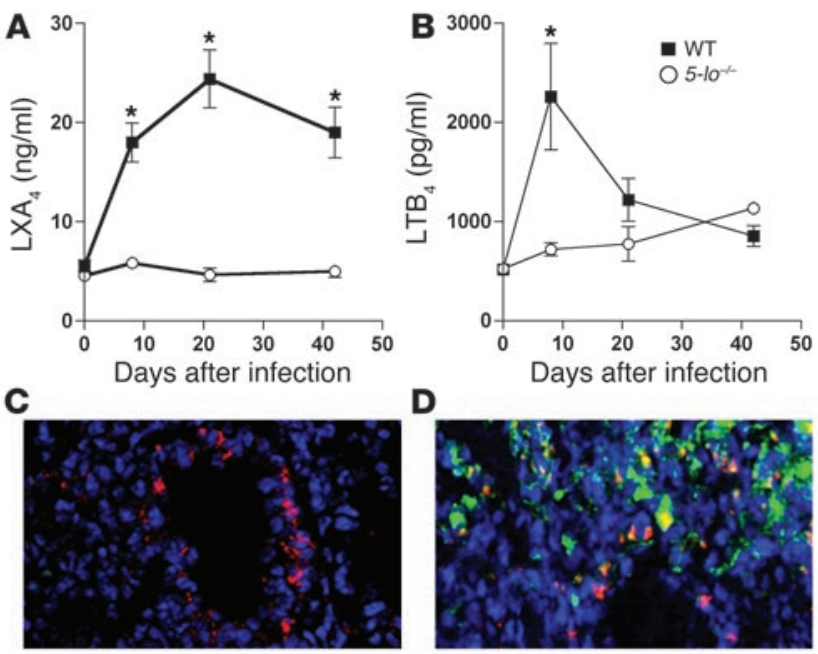

endothelial cells and macrophages provide the source of the 5-LO required for the synthesis of these eicosanoids.

5-LO-deficient mice display enhanced control of M. tuberculosis infection. To investigate the role of 5-LO in M. tuberculosis infection in vivo, we assessed bacterial burdens and tissue histopathology in 5- $l o^{-/-}$and control animals. Lungs from $5-1 o^{-/-}$mice displayed significant reductions in mycobacterial load at both 21 and 42 days after infection when compared with similarly infected WT control animals (Figure 2, A and B). Similar reductions in bacterial counts were observed in spleens from the same animals (data not shown). Acid-fast staining confirmed that fewer mycobacteria were present in the lungs of 5-LO-deficient mice compared with B6, 129S F2/J control mice (Figure 2, C and D, respectively). In addition, lungs from 5-LO-deficient mice infected for 50 days with M. tuberculosis showed dramatically reduced tissue inflammation compared with lungs from infected WT animals. Consistent with their high mycobacterial burden 50 days after infection, lungs from WT mice exhibited severe, widespread alveolitis and interstitial pneumonitis as well as areas of necrosis (Figure 3, A and B). In contrast, lungs from similarly infected 5 - $l 0^{-/-}$mice displayed much less inflammation and little evidence of tissue necrosis (Figure 3, C and D).

Consistent with their reduced bacterial load, $5-l^{-/-}$mice infected with $300 \mathrm{CFU} /$ mouse displayed enhanced survival compared with similarly infected B6, 129S F2/J mice (Figure 2E). In these experiments, WT mice succumbed to aerogenic M. tuberculosis infection more rapidly than has been reported previously in mice of this

\section{Figure 2}

Increased resistance of 5-LO-deficient mice to M. tuberculosis infection. Lungs from WT (black bars) and $5-10^{-/-}$(gray bars) mice were harvested at several time points after infection with an average of 300 (A) or 50 (B) CFU/mouse and mycobacterial burdens determined. Results are mean \pm SE of measurements from 4 animals. ${ }^{*} P<0.05$. Representative acid-fast bacilli-stained sections from lungs of 50-day-infected WT (C) and 5- $/ \mathrm{O}^{-/-}$(D) mice (300 CFU/animal) illustrate the reduction in acid-fast bacilli (red staining) in the $\mathrm{KO}$ animals. Original magnification, $\times 63$. (E) WT B6, 129S F2/J (filled symbols) and 5-LO-deficient (open symbols) animals were aerogenically infected with an average of 300 $\mathrm{CFU} / \mathrm{mouse}$ (high dose [HiD]; squares) or with $50 \mathrm{CFU} /$ mouse (low dose [LoD]; circles) ( $n=10$ animals per group) and survival monitored. The results shown are representative of 2 independent experiments performed at each dose.

\section{Figure 1}

5-LO-dependent $\mathrm{LXA}_{4}$ and $\mathrm{LTB}_{4}$ production and 5-LO expression during $M$. tuberculosis infection. WT (B6, 129J F2; filled squares) and 5-LO-deficient (B6, 129J Alox-5; open circles) animals were infected by aerosol exposure with an average of $300 \mathrm{CFU} /$ mouse of $M$. tuberculosis $\mathrm{H}_{37 R v}$ and $\mathrm{LXA}_{4}(\mathbf{A})$ and $\mathrm{LTB}_{4}(\mathbf{B})$ assessed by ELISA in serum at 8,21 and 42 days after infection. Results are mean \pm SE of measurements from 5 animals. ${ }^{*} P<0.05$ between experimental groups. Results shown are representative of 2 independent experiments. (C and D) WT lung sections were stained with anti-5-LO (red) and costained with antiF4/80 (green), followed by counterstaining with DAPI (blue). (C) 5-LO+ endothelium. (D) Several F4/80+5-LO+ cells infiltrating pulmonary tissue during M. tuberculosis infection. Original magnification, $\times 63$.

genetic strain. We reasoned that this was likely a result of the fact that the inoculum (300 CFU) was larger than that (50 CFU) used in prior studies (15). Although the extended survival of 5- $\mathrm{lo}^{-/-}$mice infected at high dose argues for their enhanced resistance, we also examined mortality at the more conventional low infectious dose (50 CFU/mouse). In this setting, both 5-LO-deficient and control mice survived at similar rates until 300 days after infection (Figure $2 \mathrm{E})$. Nevertheless, a highly significant reduction in bacterial burden similar to that observed at the higher dose of infection was evident in the lungs of these animals at days 21 and 42 (Figure 2D). Taken together, these results demonstrate that 5-LO promotes both mycobacterial growth and host susceptibility to infection.

5-LO-deficient mice infected with $M$. tuberculosis show increased expression of proinflammatory mediators. To determine whether the absence of 5-LO affects the proinflammatory responses induced by mycobacteria, we studied the time course of expression of the genes
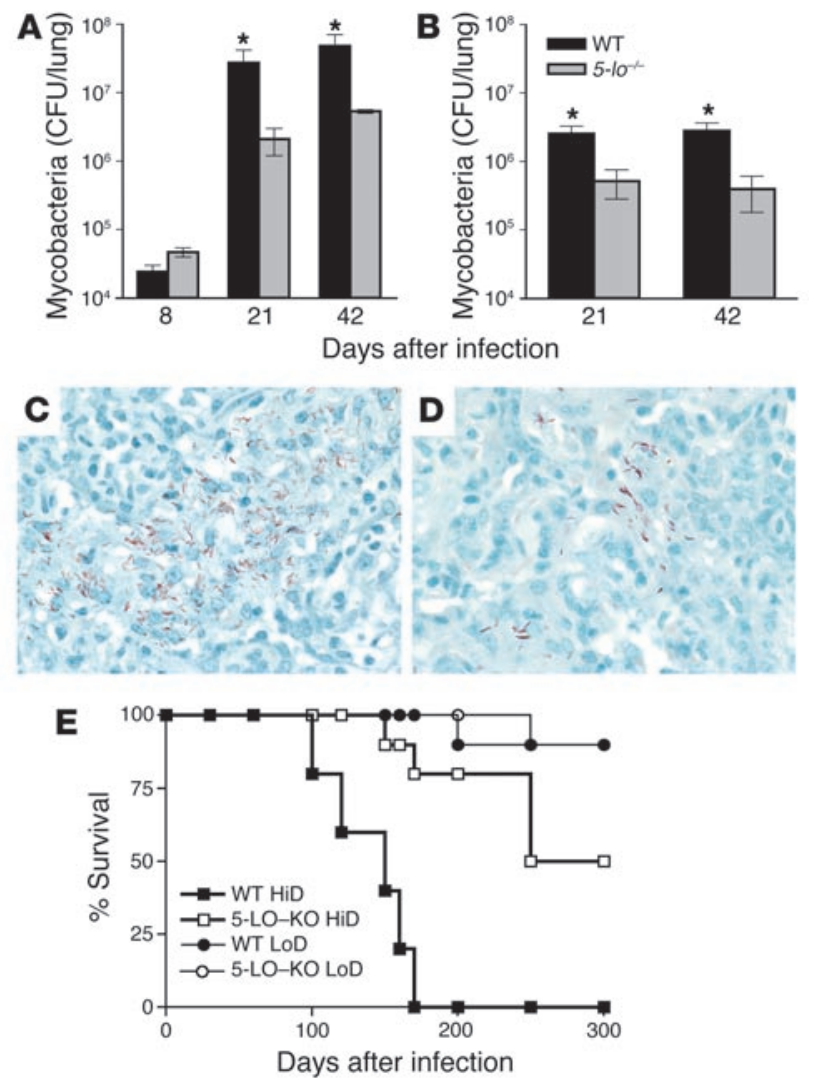


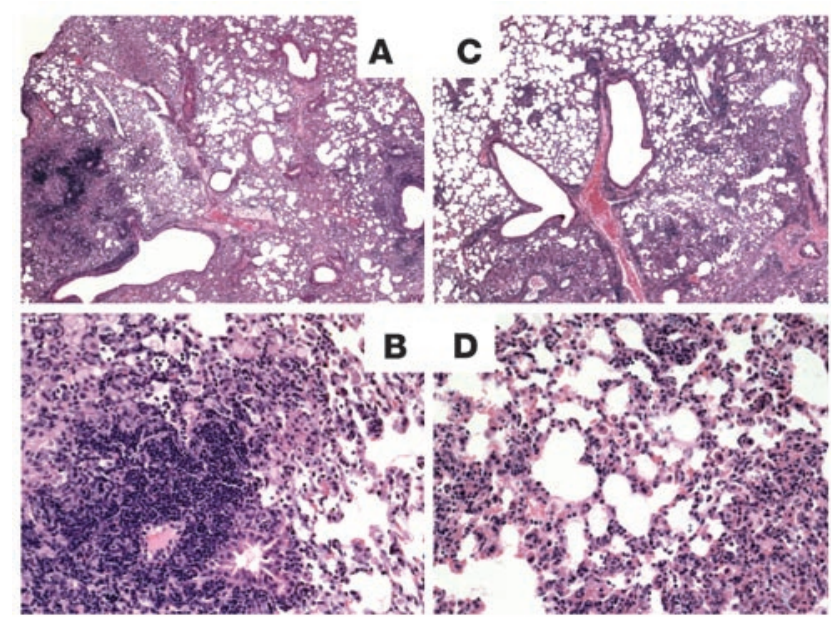

encoding IL-12, IFN- $\gamma$, TNF, and NO synthase 2 (NOS2) in the lungs of $M$. tuberculosis-infected animals. Levels of both IL-12p40 and IFN- $\gamma$ mRNA were found to be significantly elevated in infected 5-lo-/- mice compared with their WT counterparts (Figure 4, A and D), consistent with their diminished mycobacterial burdens. Despite the marked differences in pulmonary histopathology (Figure 3, A and $B$ versus $C$ and D), the levels of TNF expression in lungs did not differ significantly between infected 5- $l o^{-/-}$and WT mice (Figure 4E). Importantly, expression of the gene encoding NOS2, an enzyme required for host resistance to $M$. tuberculosis in mice (16), was found to be dramatically elevated in the absence of 5-LO at both 21 and 42 days after infection (Figure 4B). Nevertheless, no differences in IL-10 expression were observed in the lungs of $5-\mathrm{lo}^{-/-}$ versus control animals at the time intervals examined (data not shown). To confirm this gene expression data, we assayed IL-12p40 and TNF protein levels in lung homogenates from the same infected animals at day 21 after infection. As shown in Figure 4, C and F, $5-1 o^{-/-}$mice displayed significantly increased levels of IL-12p40 but not TNF. In addition, immunostaining of lung sections was performed in an attempt to identify the cellular source of the increased proinflammatory cytokine. Few CD $11 c^{+}$cells were found to coexpress IL-12p40 in lungs of WT animals (Figure 5A and Table 1), while a higher fre-

\section{Figure 4}

5-LO-deficient mice infected with $M$. tuberculosis display increased expression of proinflammatory mediators. WT and $5-1 \mathrm{O}^{-/-}$ mice were aerogenically infected (300 CFU inoculum), and relative expression of mRNAs for IL-12p40 (A), NOS2 (B), IFN- $\gamma$ (D), and TNF (E) was determined in the lungs at 8,21 and 42 days after M. tuberculosis infection. To further confirm these observations, we prepared lung homogenates from the same animal groups shown above and determined IL-12 (C) and TNF $(\mathbf{F})$ levels by ELISA. ${ }^{*} P<0.05$. The results shown are representative of 2 independent experiments. Uninf., uninfected.

\section{Figure 3}

Decreased inflammation in lungs of $M$. tuberculosis-infected 5-LOdeficient mice. Representative H\&E-stained sections of lungs from 50-day-infected (300 CFU inoculum) B6, 129S F2/J control (A and B) and 5-LO-deficient ( $\mathbf{C}$ and $\mathbf{D}$ ) animals. Note the reduction in inflammatory infiltration and greatly increased alveolar space in $5-/ 0^{-/-}$animals (C and D). Original magnification, $\times 5$ (A and C) and $\times 40$ (B and D).

quency of CD $11 \mathrm{c}^{+} \mathrm{IL}-12 \mathrm{p} 40^{+}$cells was found infiltrating the lungs of 5-LO-deficient mice (Figure 5D and Table 1). However, not all of IL-12p40 staining was associated with CD11c cells (green), which suggests that 5 -LO regulates IL-12 expression in both DCs and other leukocytes in the tissue infiltrates. In contrast, the expression of TNF and NOS2 was found to be restricted to the F4/80+ (macrophage) cell population, and although TNF expression was similar in the 2 animal groups (Figures 5, B and $\mathrm{E}$ and Table 1), the frequency of NOS $2^{+} \mathrm{F} 4 / 80^{+}$cells was dramatically enhanced in the lungs of the infected 5-LO-deficient mice (Figures 5, $\mathrm{C}$ and $\mathrm{F}$ and Table 1). These findings both agree and contrast with our previous observations on cell-associated cytokine and NOS2 expression during T. gondii infection in 5-LO-deficient mice (14). In that study, we observed enhanced IL-12 production by both CD $11 \mathrm{c}^{+}$and CD $11 \mathrm{c}^{-}$cells in brain tissue of infected 5-LO-deficient mice but failed to detect significant changes in NOS2 as reported here.

Treatment with an $\mathrm{LXA}_{4}$ analog reverses resistance in $M$. tuberculosisinfected 5-LO-deficient mice. 5-LO is involved in the biosynthesis of several eicosanoids, including $\mathrm{LXA}_{4}$, that are known to mediate local control of inflammation. Therefore, it was critical to formally establish whether the enhanced protection against M. tuberculosis infection, elevated level of type 1 cytokines, and lower mycobacterial bur-
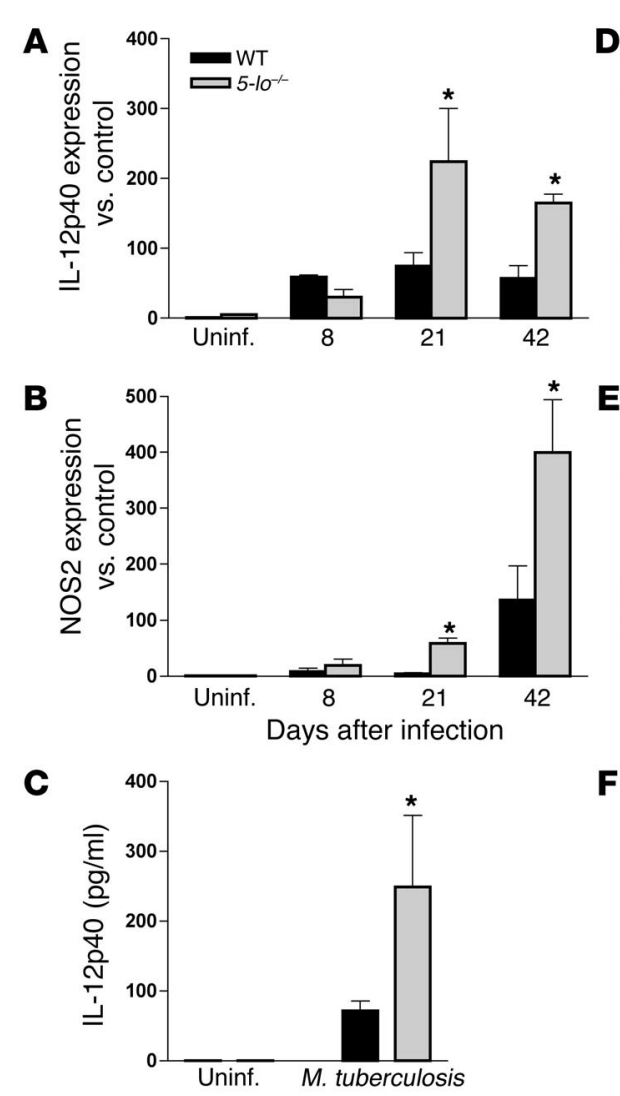
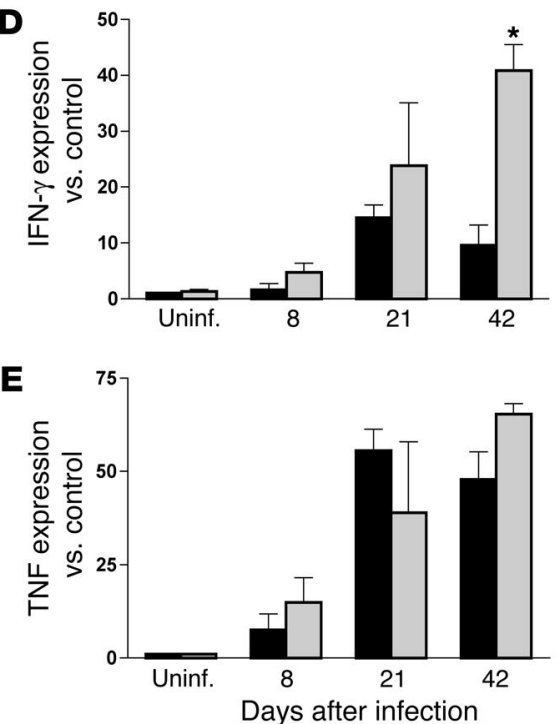

$\mathbf{F}$

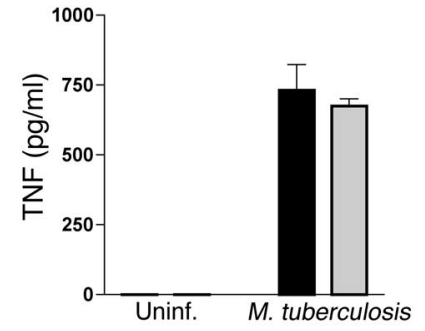



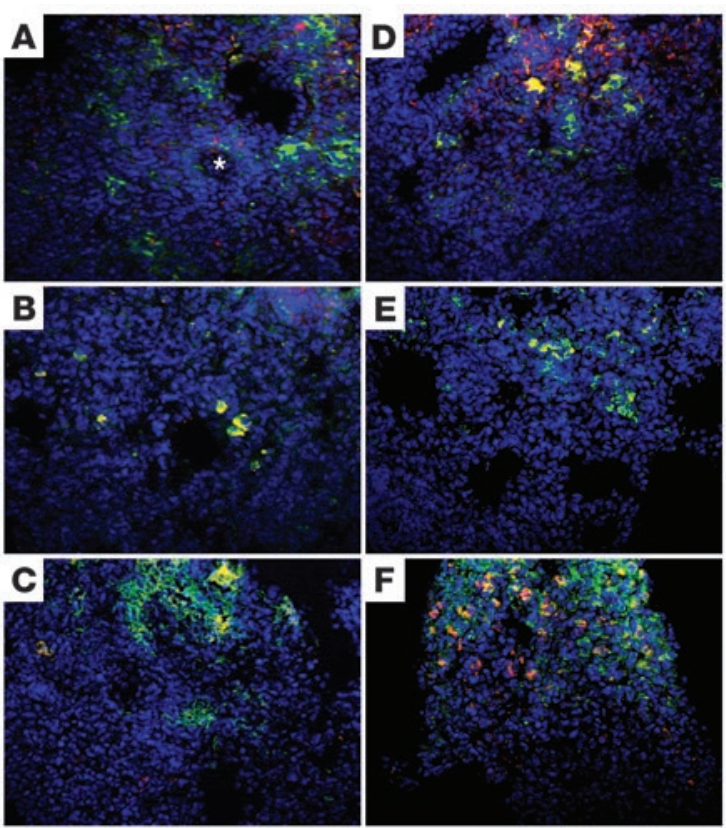

den in 5-LO-KO mice are indeed related to the absence of lipoxin generation during infection. To address this issue, we administered a stable lipoxin analog, ATLa2, to both WT controls and 5-LO-deficient mice during the first 21 days after infection. This eicosanoid analog, created by design modification of the $\omega$ end of $\mathrm{LXA}_{4}$, was shown to have increased half-life in vivo and to inhibit inflammation in several disease models (17-19). As shown in Figure 6, A and B, ATLa2 treatment abrogated the enhanced control of bacterial growth in both lungs and spleens of 5-LO-deficient mice. No alteration of in vivo resistance was noted in M. tuberculosis-infected WT controls treated with the $\mathrm{LXA}_{4}$ analog at this dose. These findings suggest that the levels of endogenous lipoxin present in the infected 5-LO-competent mice are already optimal and that additional lipoxin does not alter the host response to $M$. tuberculosis infection. When examined at 21 days after infection, the M. tuberculosis-infected 5-lo-l- mice treated with the $\mathrm{LXA}_{4}$ analog displayed a weakened Th1 response, as evidenced by reduced IFN- $\gamma$ production by splenocytes restimulated ex vivo with $M$. tuberculosis antigen (Figure 6C) but unaltered TNF levels (Figure 6D). Importantly, ATLa2 had no direct effects on mycobacterial proliferation in vitro, which argues against the possibility that the in vivo activity of this eicosanoid is due to a direct antibiotic effect (data not shown).

\section{Discussion}

Proinflammatory cytokines such as IL-12 play critical roles in the induction of host resistance to $M$. tuberculosis as well as other intracellular pathogens. These responses must be carefully regulated to avoid host tissue damage. The antiinflammatory cytokines IL-10 and TGF- $\beta$ have been implicated as key protein mediators that prevent excess IL-12, TNF- $\alpha$, and IFN- $\gamma$ production in intracellular infections. Nevertheless, these downregulatory cytokines appear

\section{Table 1}

\section{Figure 5}

Lung-infiltrating DCs and macrophages express high levels of IL-12 and NOS2 in 5-LO-deficient hosts infected with M. tuberculosis. Frozen sections of lungs from infected (300 CFU inoculum) WT (A, B, and $\mathbf{C})$ and $5-10^{-/-}(\mathbf{D}, \mathbf{E}$, and $\mathbf{F})$ mice were double stained with antiCD11c (A and D) or anti-F4/80 (B, C, E, and F) (green) and with antiIL-12p40 (A and D), anti-TNF (B and E), or anti-NOS2 (C and F) (red), then counterstained with DAPI (blue). Note the presence of many more CD $11 \mathrm{c}^{+} \mathrm{IL}-12 \mathrm{p} 40^{+}$cells and $\mathrm{F} 4 / 80^{+} \mathrm{NOS}^{+}$cells in the tissue sections from $5-10^{-1-}$ animals. The asterisk in $\mathbf{A}$ indicates the presence of multinucleated cells at the center of a granuloma. Representative micrographs (magnification, $\times 63$ ) from 4 animals per group are shown.

to have only limited effects in controlling $M$. tuberculosis replication during infection in animal models $(8,20)$. In the present study, we report evidence for the role of a novel pathway involved in dampening $M$. tuberculosis-driven proinflammatory immune responses and regulating bacterial growth that involves the 5-LO-dependent production of lipoxins.

Lipoxins such as $\mathrm{LXA}_{4}$ are biosynthesized by different cell types, including leukocytes, endothelial cells, and platelets by means of transcellular pathways (21). Recently, $\mathrm{LXA}_{4}$ was shown to have downregulatory actions on several proinflammatory mechanisms including NK cell cytotoxicity (22), leukocyte responses to proinflammatory cytokines (23), and microbial stimulation (14) as well as migration of both neutrophils (18) and eosinophils (24). Interestingly, stimulation of mucosal epithelial cells with lipoxin analogs induced the expression of a bactericidal/permeability-increasing protein, which exhibits antimicrobial activities and enables epithelial cells to engage in active microbial host defense $(25,26)$.

$\mathrm{LXA}_{4}$ dramatically reduces $T$. gondii-induced IL-12 production by DCs in vitro and by DCs as well as other cells in vivo (13, 14), which indicates a role for $\mathrm{LXA}_{4}$ in preventing uncontrolled proinflammatory responses. T. gondii triggered high levels of $\mathrm{LXA}_{4}$ $(\sim 100 \mathrm{ng} / \mathrm{ml})$ in sera of WT mice, while infected 5-LO-deficient mice produced elevated amounts of IL-12p40. Interestingly, it was recently shown that $T$. gondii synthesizes its own LO that may play a role in increasing local concentrations of $\mathrm{LXA}_{4}(27)$. In the present report, using an aerosol model of infection with $M$. tuberculosis, we also detected the induction of high levels of $\mathrm{LXA}_{4}$ as well as the leukotriene $\mathrm{LTB}_{4}$ in the sera of WT mice. However, $\mathrm{LXA}_{4}$ and $\mathrm{LTB}_{4}$ were induced with different kinetics, and only $\mathrm{LXA}_{4}$ persisted at high levels during chronic infection. Furthermore, we observed high levels of expression of 5-LO in lung endothelium and macrophages during infection. The latter results suggest that these cell populations participate in lipoxin generation in vivo and may be specifically involved in regulating local inflammatory responses during chronic experimental tuberculosis.

Higher frequency of CD11 $+\mathrm{IL}-12 \mathrm{p} 40^{+}$and $\mathrm{F} 4 / 80^{+} \mathrm{NOS2}+$ cells in lung sections from $M$. tuberculosis-infected 5-LO-deficient versus WT animals ${ }^{\mathrm{A}}$

\begin{tabular}{|c|c|c|c|c|c|}
\hline Group & CD11c+IL-12p40+ & CD11c-IL-12p40+ & NOS2+ & F4/80+TNF+ & F4/80-TNF+ \\
\hline WT & $39 \pm 6.11$ & $28.66 \pm 6.4$ & $101 \pm 19.05$ & $16.33 \pm 4.8$ & 15.77 \\
\hline $5-10^{-1-}$ & $100.16 \pm 6.63^{B}$ & $39.5 \pm 4.81$ & $182.66 \pm 13.96^{B}$ & $15.33 \pm 1.01$ & $135.66 \pm 16.44$ \\
\hline
\end{tabular}

AMicroscopic quantitation of fluorochrome-positive cells was performed on the lung sections described in Figure 5. Results are expressed as the number of positive cells per field \pm SEM determined from 10 observation fields per slide and 3 slides per mouse ( 3 animals/group). ${ }^{B} P<0.05$ vs. WT. 

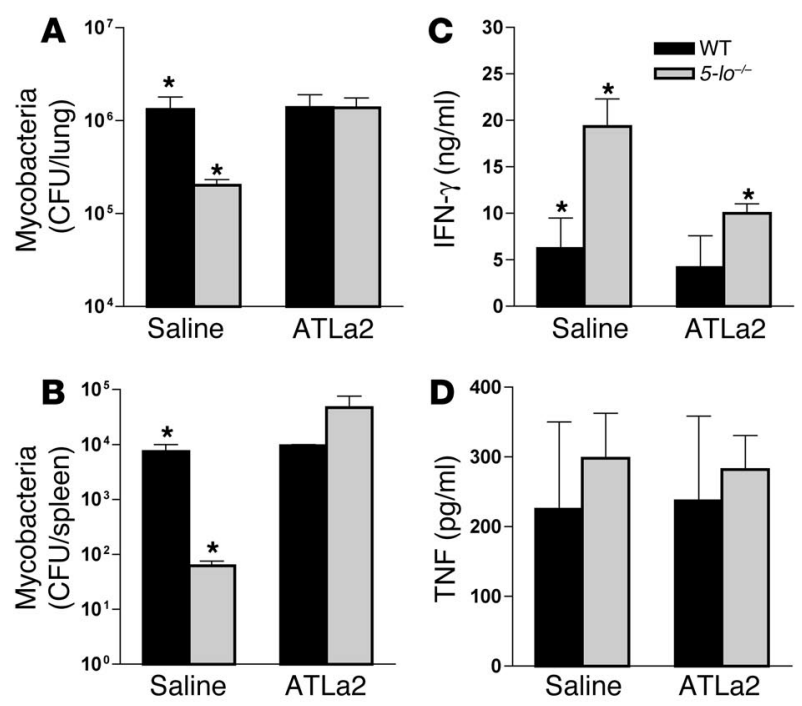

Figure 6

In vivo administration of a stable analog of $\mathrm{LXA}_{4}$ in $M$. tuberculosisinfected mice. WT or 5- $/ 0^{-/-}$mice were infected by aerosol exposure with M. tuberculosis (300 CFU inoculum) and treated 3 times a week by gavage from days 2 to 20 with vehicle or ATLa2 at $100 \mathrm{ng} / \mathrm{ani}-$ $\mathrm{mal} /$ treatment. Mice were sacrificed, and mycobacterial burdens were assessed in lungs (A) and spleens (B), 21 days after infection. Spleen cell cultures from the same animals were restimulated with purified protein derivative, and 72 hours later, IFN- $\gamma(\mathbf{C})$ and TNF (D) levels were measured in supernatants by ELISA. Results are the mean \pm SD from 5 animals per group. ${ }^{*} P<0.05$ between groups.

Whereas $T$. gondii-exposed $5-l 0^{-/-}$mice succumbed rapidly to infection, the reduced lipoxin generation in 5- $l 0^{-/-}$mice infected with $M$. tuberculosis was associated with enhanced survival at highdose aerosol challenge, although there were no apparent differences in mortality at the lower inoculum infections. These contrasting outcomes of the 2 infection models may stem from differences in the intrinsic virulence and immune-stimulatory properties of the pathogens in question. T. gondii is a highly virulent and fast-replicating microorganism that requires the induction of a potent immune response to protect the host and produce chronic persistent infections necessary for promoting its transmission. M. tuberculosis, while also inducing latent infections, replicates slowly and, at least in the mouse model, induces a weaker Th1 response than does $T$. gondii. Hence, in the absence of lipoxin-mediated counterregulation, the ensuing cellular responses are enhanced, triggering immunopathology and mortality in T. gondii infection, whereas in M. tuberculosis infection, this enhancement results in increased control of bacterial replication. The observed restriction in mycobacterial growth does not appear to be complete, however, since high-dose M. tuberculosis-infected 5- $l 0^{-/-}$mice eventually began to succumb at 150 days after infection, and this mortality is associated with an approximate log increase in mycobacterial load compared with earlier time points (e.g., day 42) (data not shown). Whether the late death of the infected 5-LO-deficient animals is due solely to increased bacterial burden remains unclear.

Since 5-LO is required for both leukotriene and lipoxin biosynthesis, reconstitution experiments were performed to more directly assess the role of the latter group of eicosanoids in the regulation of mycobacterial growth in vivo. Importantly, administration of the stable lipoxin analog ATLa2 to M. tuberculosis-infected 5-lo-/mice, restored both pulmonary mycobacterial loads and IFN- $\gamma$ production by purified protein derivative-stimulated splenocytes to levels comparable to those observed in infected WT animals. Although this observation does not rule out the possible participation of other 5-LO-dependent mediators, it demonstrates that a deficiency in lipoxins is sufficient to explain the effects on bacterial growth and host response seen in the infected 5-LO-deficient animals. ATLa2 treatment has previously been shown to reduce inflammatory cell infiltration in a number of different disease models (17-19). Although this subject is not directly addressed in the present article, it is probable that the observed effects of ATLa2 reconstitution in our experiments result from decreased effector cell recruitment into infected lung.

In summary, our findings demonstrate the existence of a novel pathway involved in controlling proinflammatory and Th1 immune responses against $M$. tuberculosis infection in vivo via the generation of 5-LO-dependent lipoxin formation. These observations suggest that the regulation of lipoxin biosynthesis merits further investigation as a potential immunopharmacologic intervention for enhancing the control of mycobacterial replication in tuberculosis patients. In this regard, it should be noted that 5-LO inhibitors are already in clinical trial for asthma, and therefore it may be possible to rapidly design and implement a study testing the efficacy of this strategy for intervention in tuberculosis (28-30).

\section{Methods}

Mice. WT controls (B6, 129S F2/J) and 5-LO-deficient (B6, 129S Alox-5, F2/J) mice were obtained from The Jackson Laboratory and were bred and maintained in an NIAID Association for the Assessment and Accreditation of Laboratory Animal Care-accredited animal facility. Female animals 5-8 weeks old were used in all experiments. All experiments were approved by the NIAID Institutional Animal Care and Use Committee.

M. tuberculosis infection. The M. tuberculosis $\mathrm{H} 37 \mathrm{Rv}$ strain was passaged through mice, grown in culture once, and frozen in aliquots. Prior to infection, an aliquot was thawed, diluted in PBS, and briefly sonicated in a cuphorn sonicator. Mice were placed in a closed, nose-only aerosolization system (CH Technologies) and exposed for 15 minutes to nebulized M. tuberculosis. Two different bacterial doses were employed: 50 and $300 \mathrm{CFU}$ per mouse. To assess mycobacterial load, we harvested lungs and spleens at different times after infection, and tissue homogenates were diluted in buffered saline and cultured on 7H11 agar plates. Colony counts were determined 21 days later.

Histopathology. Lung and spleen tissues were harvested, fixed with neutral buffered formalin, and paraffin embedded. Serial sections were stained with H\&E for histopathologic analysis or with Kinyoun's acid fast stain for in situ detection of mycobacteria.

Eicosanoid and cytokine determinations. Serum levels of eicosanoids and cytokines were measured using commercial ELISA kits obtained from Neogen Corp. $\left(\mathrm{LXA}_{4}\right)$, Cayman Chemical Co. $\left(\mathrm{LTB}_{4}\right)$, and R\&D Systems, (IL-12p40, TNF, IFN- $\gamma$ ). For cytokine detection in lungs, tissue was homogenized and centrifuged at $300 \mathrm{~g}$ for 7 minutes. Supernatants aliquots were frozen at $-80^{\circ} \mathrm{C}$ for later analysis by ELISA.

Measurement of gene expression in lung. Total RNA was isolated from lungs and real-time RT-PCR was performed on an ABI Prism 7900 sequence detection system (Applied Biosystems) using SYBR Green PCR Master Mix (Applied Biosystems) after reverse transcription of $1 \mu \mathrm{g}$ RNA using Superscript II reverse transcriptase (Invitrogen Corp.). The relative level of gene expression was determined by the comparative $\mathrm{Ct}$ method as described by the manufacturer, whereby data for each sample were normalized to hypoxanthine phospho-ribosyl-transferase (hprt) and expressed as a fold change compared 
with untreated controls. The following primer pairs were used: for hprt, GTTGGTTACAGGCCAGACTTTGTTG (forward) and GAGGGTAGGCTGGCCTATAGGCT (reverse); il-12p40, CTCACATCTGCTGCTCCACAAG (forward) and AATTTGGTGCTTCACACTTCAGG (reverse); if $n-\gamma$, AGAGCCAGATTATCTCTTTCTACCTCAG (forward) and CTTTTTTCGCCTTGCTGCTG (reverse); nos2, TGCCCCTTCAATGGTTGGTA (forward) and ACTGGAGGGACCAGCCAAAT (reverse); tnf,AAAATTCGAGTGACAAGCCTGTAG (forward) and CCCTTGAAGAGAACCTGGGAGTAG (reverse).

In situ staining. In situ immunostaining of CD11c, F4/80, 5-LO, IL-12p40, TNF, and NOS2 was performed as previously described (14). In brief, acetone-fixed, frozen sections were incubated with biotin-conjugated antibodies against $\mathrm{CD} 11 \mathrm{c}$ or F4/80 (BD). After washing, sections were exposed to streptavidin-conjugated Alexa Fluor 486 (Invitrogen Corp.). Sections were simultaneously double stained with rabbit anti-IL-12p40, anti-TNF, anti-5-LO, or anti-NOS2 pAb, and the reaction was developed with antirabbit IgG Alexa Fluor 594 (Invitrogen Corp.), followed by counterstaining with DAPI (Invitrogen Corp.). After washing, the sections were examined microscopically, and the images were recorded using the ApoTome system (Carl Zeiss Microimaging, Inc.).

In vivo lipoxin analog treatment. ATLa2, 15-epi-16-phenoxy-parafluoro$\mathrm{LXA}_{4}$-methyl ester (a generous gift from J. Parkinson, Berlex Biosciences, Richmond, California, USA), was used in vivo as a stable lipoxin analog as previously reported (18). It was administered by gavage 3 times a week at a dose of $0.2 \mathrm{ml}$ (100 ng/animal/treatment) as previously described (19). Treatment was started 2 days after infection and continued until day 20. Similarly infected control mice were likewise treated with vehicle alone.
Spleen cell cultures. Spleens from M. tuberculosis-infected mice were disaggregated through $40-\mu \mathrm{m}$ cell strainers, and red blood cells were lysed osmotically. Splenocytes $\left(5 \times 10^{6}\right.$ cells $\left./ \mathrm{ml}\right)$ in RPMI 1640 medium (Invitrogen Corp.) supplemented with 10\% fetal calf serum (HyClone), $10 \mathrm{mM}$ HEPES (Invitrogen Corp.), $2 \mathrm{mM}$ glutamine (Invitrogen Corp.), $100 \mathrm{U} / \mathrm{ml}$ penicillin, $100 \mathrm{~g} / \mathrm{ml}$ streptomycin (Invitrogen Corp.), and $5.5 \times 10^{-5} \mathrm{M} 2$-mercaptoethanol (Invitrogen Corp.) were distributed in 96-well plates and stimulated with $10 \mu \mathrm{g} / \mathrm{ml}$ of purified protein derivative (Statens Serum Institut). After 72 hours at $37^{\circ} \mathrm{C}$ with $5 \% \mathrm{CO}_{2}$ atmosphere, supernatants were collected for determination of IFN- $\gamma$ levels.

Statistical analysis. Statistical significance was assessed by unpaired Student's $t$ test (parametric) or Mann-Whitney $U$ test (nonparametric), and $P<0.05$ was considered significant.

\section{Acknowledgments}

We are grateful to Jose Ribeiro and Warwick Britton for their helpful discussions and criticism.

Received for publication November 11, 2004, and accepted in revised form March 29, 2005.

Address correspondence to: Julio Aliberti, Department of Immunology, Duke University Medical School, 136 Jones Building, Box 3010, Duke University Medical Center, Durham, North Carolina 27710, USA. Phone: (919) 613-7833; Fax: (919) 684-8982; E-mail: julio.aliberti@duke.edu.
1. Cooper, A.M., et al. 1995. The role of interleukin-12 in acquired immunity to Mycobacterium tuberculosis infection. Immunology. 84:423-432.

2. Flynn, J.L., et al. 1995. Tumor necrosis factor-alpha is required in the protective immune response against Mycobacterium tuberculosis in mice. Immunity. 2:561-572

3. Flynn, J.L., et al. 1995. IL-12 increases resistance of BALB/c mice to Mycobacterium tuberculosis infection. J. Immunol. 155:2515-2524.

4. de Jong, R., et al. 1998. Severe mycobacterial and Salmonella infections in interleukin-12 receptordeficient patients. Science. 280:1435-1438.

5. Altare, F., et al. 1998. Impairment of mycobacterial immunity in human interleukin-12 receptor deficiency. Science. 280:1432-1435.

6. Jouanguy, E., et al. 1996. Interferon-gammareceptor deficiency in an infant with fatal bacille Calmette-Guerin infection. N. Engl. J. Med. 335:1956-1961.

7. Keane, J., et al. 2001. Tuberculosis associated with infliximab, a tumor necrosis factor alpha-neutralizing agent. N. Engl. J. Med. 345:1098-1104.

8. Jung, Y.J., Ryan, L., LaCourse, R., and North, R.J. 2003. Increased interleukin-10 expression is not responsible for failure of $\mathrm{T}$ helper 1 immunity to resolve airborne Mycobacterium tuberculosis infection in mice. Immunology. 109:295-299.

9. Roach, D.R., et al. 2001. Endogenous inhibition of antimycobacterial immunity by IL-10 varies between mycobacterial species. Scand. J. Immunol. 54:163-170

10. Jung, Y.J., LaCourse, R., Ryan, L., and North, R.J. 2002. Evidence inconsistent with a negative influence of $\mathrm{T}$ helper 2 cells on protection afforded by a dominant T helper 1 response against Mycobacterium tuberculosis lung infection in mice. Infect. Immun. 70:6436-6443.

11. Goldfeld, A.E. 2004. Genetic susceptibility to pulmonary tuberculosis in Cambodia. Tuberculosis (Edinb.). 84:76-81.

12. Aliberti, J. 2005. Host persistence: exploitation of anti-inflammatory pathways by Toxoplasma gondii. Nat. Rev. Immunol. 5:162-170.

13. Aliberti, J., Hieny, S., Reis e Sousa, C., Serhan, C.N., and Sher, A. 2002. Lipoxin-mediated inhibition of IL-12 production by DCs: a mechanism for regulation of microbial immunity. Nat. Immunol. 3:76-82.

14. Aliberti, J., Serhan, C., and Sher, A. 2002. Parasite-induced lipoxin A4 is an endogenous regulator of IL-12 production and immunopathology in Toxoplasma gondii infection. J. Exp. Med. 196:1253-1262.

15. Scanga, C.A., et al. 2004. MyD88-deficient mice display a profound loss in resistance to Mycobacterium tuberculosis associated with partially impaired Th1 cytokine and nitric oxide synthase 2 expression. Infect. Immun. 72:2400-2404.

16. MacMicking, J.D., et al. 1997. Identification of nitric oxide synthase as a protective locus against tuberculosis. Proc. Natl. Acad. Sci. U. S. A. 94:5243-5248.

17. Devchand, P.R., et al. 2005. A synthetic eicosanoid LX-mimetic unravels host-donor interactions in allogeneic BMT-induced GvHD to reveal an early protective role for host neutrophils. FASEB J. 19:203-210.

18. Clish, C.B., et al. 1999. Local and systemic delivery of a stable aspirin-triggered lipoxin prevents neutrophil recruitment in vivo. Proc. Natl. Acad. Sci. U. S. A. 96:8247-8252.

19. Bannenberg, G., et al. 2004. Lipoxins and novel 15-epi-lipoxin analogs display potent anti-inflammatory actions after oral administration. Br. J. Pharmacol. 143:43-52.

20. Dai, G., and McMurray, D.N. 1999. Effects of modulating TGF-beta 1 on immune responses to mycobacterial infection in guinea pigs. Tuber. Lung Dis. 79:207-214.

21. Serhan, C.N. 2002. Lipoxins and aspirin-triggered 15-epi-lipoxin biosynthesis: an update and role in anti-inflammation and pro-resolution [review]. Prostaglandins Other Lipid Mediat. 68-69:433-455.

22. Ramstedt, U., Ng, J., Wigzell, H., Serhan, C.N., and
Samuelsson, B. 1985. Action of novel eicosanoids lipoxin A and B on human natural killer cell cytotoxicity: effects on intracellular cAMP and target cell binding. J. Immunol. 135:3434-3438.

23. Hachicha, M., Pouliot, M., Petasis, N.A., and Serhan, C.N. 1999. Lipoxin (LX)A4 and aspirin-triggered 15-epi-LXA4 inhibit tumor necrosis factor 1alpha-initiated neutrophil responses and trafficking: regulators of a cytokine-chemokine axis. J. Exp. Med. 189:1923-1930

24. Bandeira-Melo, C., et al. 2000. Cutting edge: lipoxin (LX) A4 and aspirin-triggered 15-epi-LXA4 block allergen-induced eosinophil trafficking. J. Immunol. 164:2267-2271.

25. Canny, G., et al. 2002. Lipid mediator-induced expression of bactericidal/ permeability-increasing protein (BPI) in human mucosal epithelia. Proc. Natl. Acad. Sci. U. S. A. 99:3902-3907.

26. Levy, O., Canny, G., Serhan, C.N., and Colgan, S.P. 2003. Expression of BPI (bactericidal/permeabilityincreasing protein) in human mucosal epithelia. Biochem. Soc. Trans. 31:795-800.

27. Bannenberg, G.L., Aliberti, J., Hong, S., Sher, A., and Serhan, C. 2004. Exogenous pathogen and plant 15-lipoxygenase initiate endogenous lipoxin A4 biosynthesis. J. Exp. Med. 199:515-523.

28. Israel, E., Cohn, J., Dube, L., and Drazen, J.M. 1996. Effect of treatment with zileuton, a 5-lipoxygenase inhibitor, in patients with asthma. A randomized controlled trial. Zileuton Clinical Trial Group. JAMA. 275:931-936.

29. DuBuske, L.M., Grossman, J., Dube, L.M., Swanson, L.J., and Lancaster, J.F. 1997. Randomized trial of zileuton in patients with moderate asthma: effect of reduced dosing frequency and amounts on pulmonary function and asthma symptoms. Zileuton Study Group. Am. J. Manag. Care. 3:633-640.

30. Schwartz, H.J., Petty, T., Dube, L.M., Swanson, L.J., and Lancaster, J.F. 1998. A randomized controlled trial comparing zileuton with theophylline in moderate asthma. The Zileuton Study Group. Arch. Intern. Med. 158:141-148. 